\title{
E-SERVICES FOR VIRTUAL ENTERPRISE BROKERAGE
}

\author{
Ricardo Mejía, Joaquín Aca, Eunice García, Arturo Molina \\ CSIM-ITESM \\ Ave. Eugenio Garza Sada 2501 Sur \\ Monterrey, N.L. 64849 Mexico \\ rmejia@tamayo.mty.itesm.mx, jaca@tamayo.mty.itesm.mx \\ iegarcia@tamayo.mty.itesm.mx, armolina@campus.mty.itesm.mx
}

\begin{abstract}
Nowadays the global competitive environment has special impact on Virtual Business development. A special issue related to the exploitation of global business opportunities is the role played by Information Technologies in Virtual Enterprises. There have been several e-application developments to support key processes of a Virtual Enterprise Broker (VEB). However, it is important to design and develop not only e-applications, but also e-Services that are easy to implement and have an impact in the performance of VEB activities. This paper describes a methodology to evaluate, structure and implement e-Services in order to assure that these applications will improve the operations of the VE. These e-services will demonstrate how their implementation improve the performance of the VEB activities in the creation of Integrated Supply Chains between Small and Medium Enterprises (members of Virtual Industry Clusters) to OEMs (Original Equipment Manufacturers).
\end{abstract}

\section{INTRODUCTION}

In the actual global competitive environment, big companies are taking more care about their sourcing strategies and they are demanding quality and shorter cycle times at the best price. Therefore, the Virtual Enterprise Broker (VEB) must improve its process of business opportunities exploitation, in order to take advantage of the huge amount of opportunities that global sourcing might offer. These aspects are very important not only for mass production but also for special projects or product outsourcing to potential suppliers. Large firms like, OEM and Maquiladoras ${ }^{1}$ offer to the VEB the challenge to explore new strategies and redefine how new business can be exploited.

A VEB must use available resources from partners to form a business entity by means of network communication technology. The Information Technology enlarges the working space to globalization and sets up a new type of enterprise through Internet. The change of organization form requires the corresponding

\footnotetext{
${ }^{1}$ Maquiladoras are international manufacturing companies established in other countries with special taxes agreements.
} 
management technology, thus it should seek a new management model and technology to fit the implementation of Virtual Enterprises (VE).

The supportive role of Information Technology for handling information is particularly important because nothing else (people, parts, products, machines) can act if information is not accessible to make decisions. To the extent that information should be handled in a efficient and effective manner in order to allow the flows of inputs into, through and out of manufacturing systems be improved (Gunasekaran and McGaughey, 2002). Information Technologies can optimize and support the core processes carried out by the VEB in order to fulfill customer needs through the creation of Virtual Enterprises. VE will be the efficient organization form in the future, due to the rapid development of computer techniques and information technology (Xu et. al., 2002).

\section{VIRTUAL ENTERPRISE BROKER CORE PROCESS}

The Virtual Enterprise Broker (VEB) performs an important role in virtual enterprises development, being the entity responsible of create, manage and dissolve a VE in order to exploit global business opportunities. The activities carried out by the VEB through its Core Processes are very important for the fulfillment of customer requirements. Therefore, the creation of new tools to support VEB activities is an important area of research in order to improve the VE performance. Four core processes have been defined to support VEB activities (Mejía and Molina, 2002): Analysis of Market Requirements, Project Planning, Project execution and Customer Service (Follow-up). The main tasks carried out by the VEB can be analyzed through the Virtual Enterprise Life Cycle that is divided into five main phases: Identification, Formation, Design, Operation and Dissolution (Kanet et. al., 1999). The broker in a VE is foremost a member of the enterprise. It acts as an information broker, an initiator of business by recognizing new opportunities and exploiting them through searching and choosing appropriate, competent and complementary partners. It is the coordinator of the VE and moderator during the execution/operation of the VE's mission and is the primary point of contact for customers of the VE. Information Technologies are necessary to support key activities related to the VEB processes. Furthermore, the process of creating VE's is composed of tasks that can be facilitated by specific computer applications in order to achieve the automation of some of the VEB core processes.

In the Virtual Industry Clusters (VIC, 2001) there have been several eapplication developments to support the activities carried out by the Virtual Enterprise Broker (VEB). Those e-applications support VEB activities in order to have the right information, at the right moment, in the right place. Some applications are: Enterprise Core Competence Database, Enterprise Capability Broker Matrix and Broker Capacity Planning Tool (Mejía and Molina, 2002). All these applications are computer based tools, but are not e-services, as shown in figure 1. In our research, an e-service is the integration of e-applications to offer a brokerage service by the VEB. 


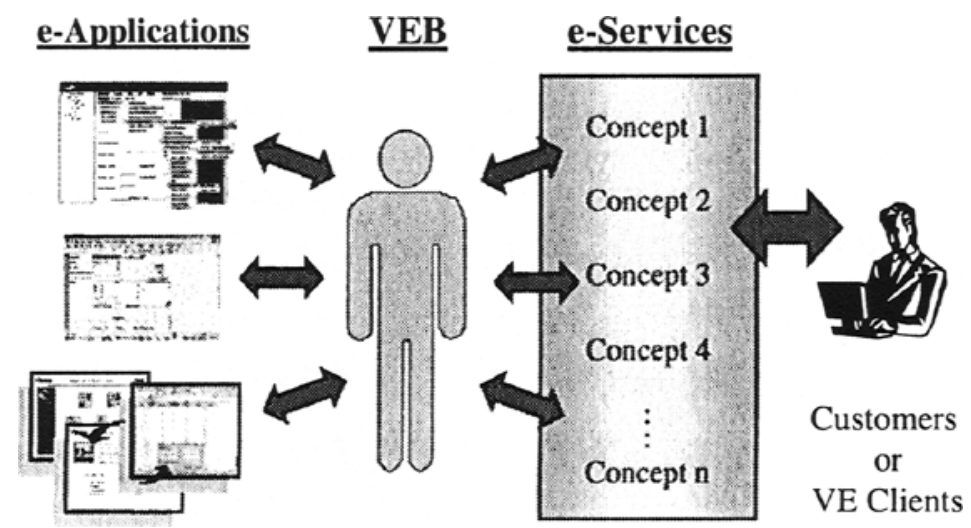

Figure 1 - e-Applications and e-Services concepts

\section{METHODOLOGY}

The methodology described here allows the VEB to evaluate and design different e-services in a systematic form, in order to implement those e-services with high potential to improve VE performance.

\subsection{Conceptualization}

This phase is focused on Brainstorming for potential e-services, where concepts generated at this stage must be classified in five categories:

a) Information. It implies information, as laws, news, technological innovations, etc. Its implementation is very easy with a low business impact. It is a time depending tool, requiring actualization over the time.

b) Community. It implies interaction with experts or lead users of different industries in order to share experiences to take advantage in productive process. It is easy to implement and medium impact in the business.

c) Directories. It implies access to manufacturers and providers databases. It is medium difficulty implementation and impact business.

d) Collaboration. It implies the dynamic interaction between parties of the project in order to manage it. It is difficult to implement and it has medium to high impact in the business.

e) Process facilitation. It implies the automation of key activities using a specific technological tool. This is very difficult to implement, but it has high impact.

It is necessary to decide which concepts are going to continue through the evaluation process. Depending on the actual organization needs, the broker use two main criteria to decide which idea is going to be the most feasible: "Easy implementation" and "business impact", based on the Cisco Methodology "Net Ready" (Hartman and Sifonis, 2000).

\subsection{Evaluation}

All brainstormed e-services within a chosen category are evaluated individually through the Cisco Methodology again at this stage. The results should be reflected in a graph like in Figure 2. In this case Concept 4 is considered as "quick wins" and 
Concept 5 is considered as "hanging fruit". A concept is selected and the next step is evaluating the concept regard two variables:

- Process issues: Cost effects, Optimize channel structure, National coverage, Channel fragmentation reduction and Partner integration.

- Economical issues: Superior service, Business support, Time reduction cycle and Knowledge / cluster intelligence.

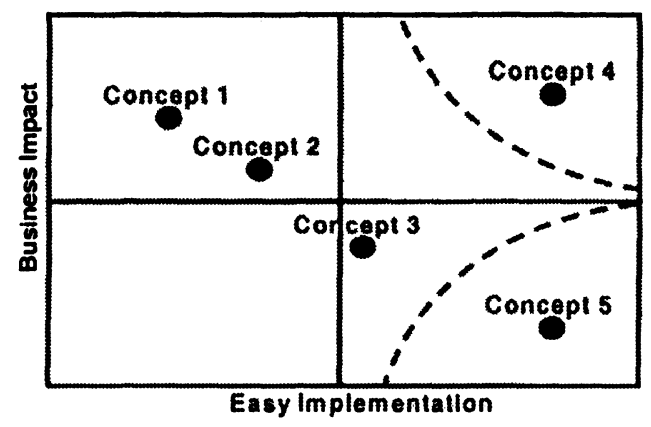

Figure 2 - Evaluation graph for e-services concepts

\subsection{Process Modeling}

An e-service is the automation of a given methodology or process. Depending on the level of detail, the modeling of the process is going to be more complex, and is important for the application developer to achieve the necessary level in order to design an effective e-service. In our research the VEB processes are structured but not detailed. The experts are in charge of modeling and structuring VEB processes in order to design an automated application. The process modeling has two levels: a) first level defines the activities, deliverables and resources of the process, the result is a list process; b) second level is the flow diagram for each process (see Figure 3).

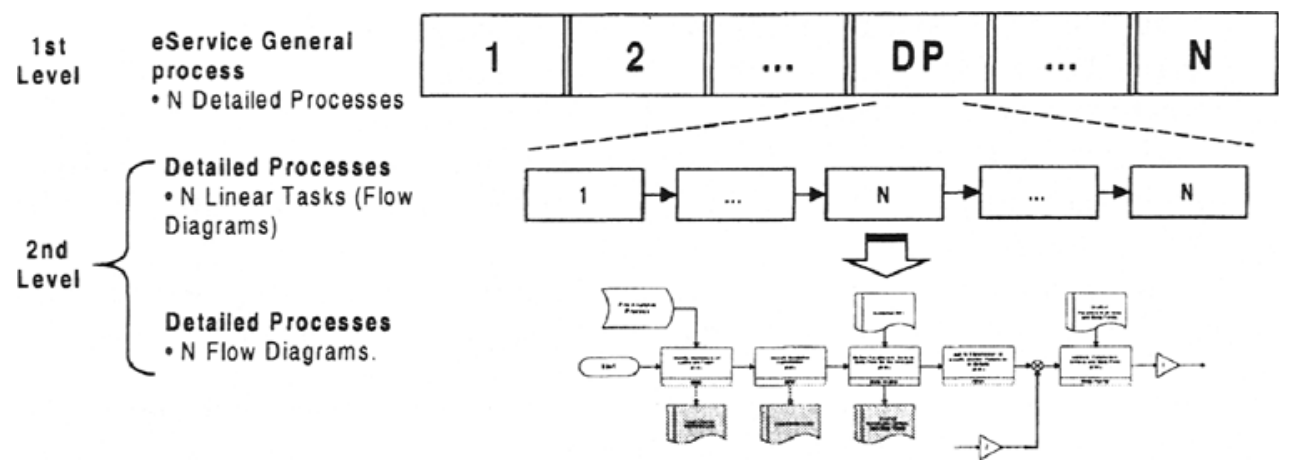

Figure 3 - e-service process modeling

\subsection{Applications Selection}

This stage is the identification, evaluation and selection of e-application vendors. The application must be represented in functional structure in order to evaluate performance functionality for each vendor. The valuation matrix and valuation symbols are show in Figure 4. 


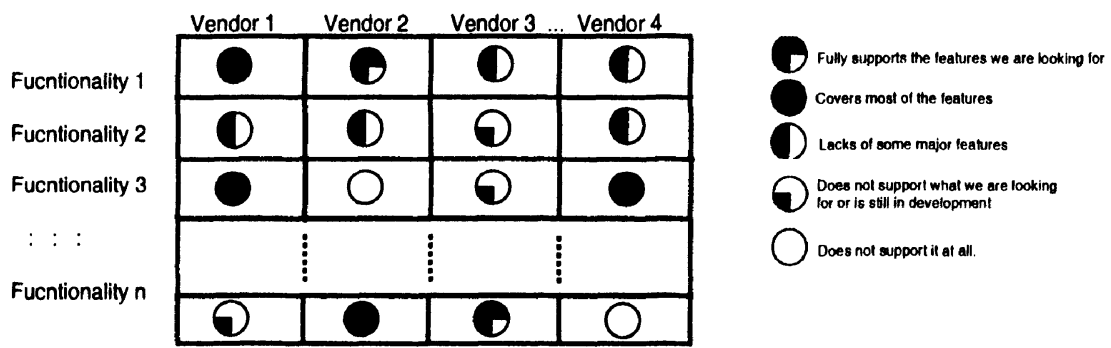

Figure 4 - Matrix valuation and valuation symbols

\subsection{Infrastructure Design}

Detailed descriptions of the technological requirements of the e-application are selected at this stage. The technical and economical feasibility is checked until technical diagrams are done. The infrastructure design is the architecture definition, and some metrics for the environment must be considered:

- Technology: Supports latest standards?, is a proven solution?, differentiates the product from other vendors?, is it robust?, allows fail-over, redundancy?, supports major OS platforms?

- Integration: Supports open standards?, is an integrated solution?, can the product be easily connected with other systems?, is it easy to migrate? Has the product support from middleware vendors?, can we easily connect it with other modules of the architecture?

- Scalability: Is the product designed for a scalable scenario?, does it support clustering?, has a record of proven scalable solutions?, is it recognized in the market as a scalable solution?

- Support of package applications: Is it easy to integrate new packaged applications?, do major vendors support the product?, has a proven record of incorporating packaged applications?

- Development Tools, time to market (TTM): Has development tools designed specially for the product?, is it easy to develop new applications with the product using the existing tools?, development cycle reduces time to market?, requires high technical skills?, are the development tools integrated among them?, and with the product?

\subsection{Roadmap Implementation}

The last stage of the methodology is the implementation of the e-application. The tasks covered in this process are:

- Verification Test: Stand-alone installation verification and the Interfaces installation must be done.

- Users Acceptance Test: LAB installation and verification, Building all possible scenarios and running by key users.

- Beta Test (Soft launch): Some enterprises are selected for Beta Test mode. The employees are trained and ran a pilot event. Logistic arrangements are made and the last step is the invitation to all enterprises.

- Go-Live: Intensive technical support at the first weeks, Extensive technical support during the next weeks, continuous remote and physical assistance per request, performance management tracking \& report. 


\section{CASE STUDY}

Using the methodology described above, a list of new concepts and ideas was generated in order to select those e-services with high potential into the VE environment considering the Mexican SME characteristics. However, the time for implementation depends on the complexity of the applications to be developed and if it is systems configuration or new development. From that list, four e-services were selected through the proposed methodology in order to be designed and implemented for the brokerage activities in the Virtual Industry Cluster. Two eservices were related to "Process facilitation e-services": e-RFQ and e-selection; and two for "Collaboration e-services": e-inspection and e-fax communication.

\section{1 e-RFQ (Electronic Request for Quotation):}

Time and accuracy can define a new business opportunity, needing an application that reduces quotation time through automated quotation services over a web-based tool. These tools reduce administrative time spent on creating and issuing manual RFQ's and negotiating with suppliers using phone calls, faxes and face-toface meetings.

Quotation knowledge automation is needed, allowing customers to fill standard RFQ or specially products or process RFQ. That process is shown in Figure 5, where the standard RFQ goes trough a module specially designed in order to receive an automated quotation. The special products RFQ must go through the VEB, and then distributed among evaluated and selected cluster members. Then, the VEB send the quotation to the client. However the Client-Broker communication is still optimized.

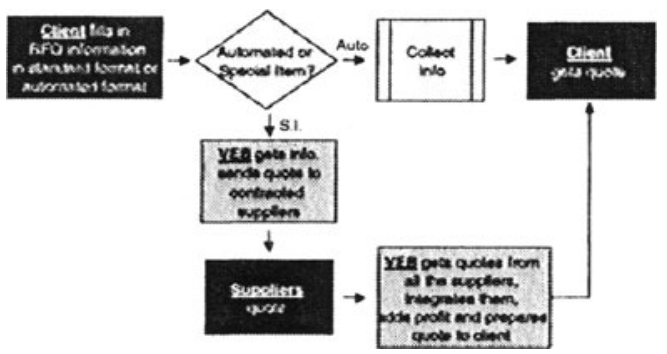

Figure 5 - e-RFQ Process Map

\section{2 e-Selection:}

One of the most important activities carried out by the VEB is the partner selection. The companies involved in the project execution are the direct responsible of the product manufacture obligating the VEB to consider, from the first stages of its Core Process, the potential players to be evaluated in order to satisfy customer requirements.

Thereby the VEB needs a technological enabler with the capability to identify, select, communicate and establish a formal agreement among the partners involved in a specific project. Also allow partners ranking for future players selections based on previous performance. Four main processes were defined for the players selection service, as shown in Figure 6. 


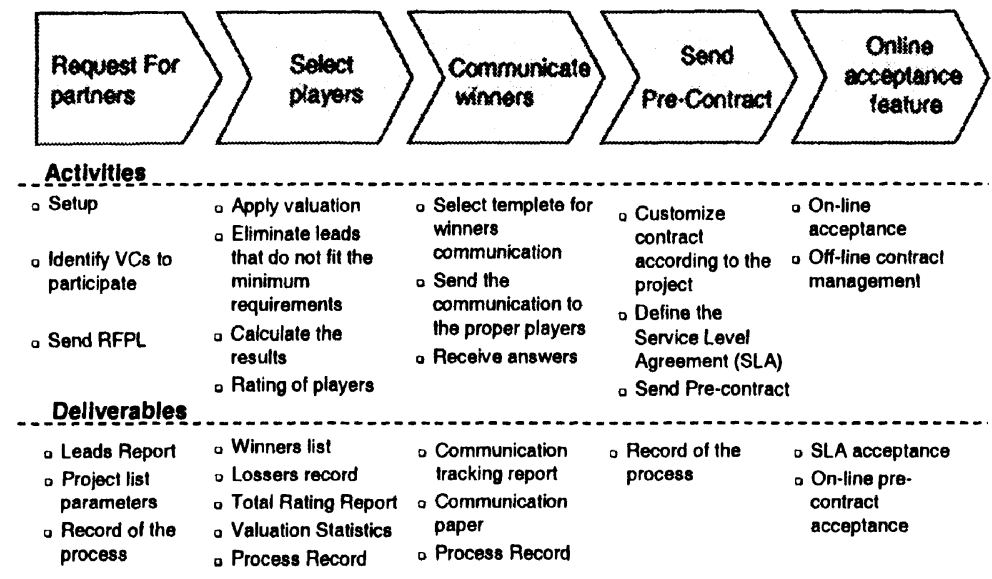

Figure 6-e-Selection main processes

\section{3 e-Inspection:}

It is common to have foreign customers, where the auditing costs are high because the travel expenses. To allow customers to follow-up their products, a web-based service is proposed trough a video camera to visually inspect products, as they are being created and tested in the supplier's factory.

Virtual Inspection will be available to customers through a web interface providing virtual auditing sessions with a Web-cam based tool. The remote interviewing between customers and Mexican Small and Medium companies (SME's) provides: A) Customer experience related to inspection, B) Customer travel time \& expenses decreasing and C) Reduction in customer notification period (Cycle time reducing)

\section{4 e-Fax Communication:}

One of the most common problems for Virtual Industry Clusters Management is the lack of Internet culture. The micro and small industries are tended to conventional communication services for doing business, as face-to-Face, Telephone and Fax.

The proposed service will act like a bridge between the "online" and the "traditional telephony" worlds, by allowing the conversion of web-generated information requests into faxes. This application will promote the e-culture in the SME's working as a warning that an important e-mail arrives, due to the fact that SME's owners will receive simultaneously a Fax with the information on the e-mail.

This e-service is easy to implement because it is a low cost communication solution by assembling "off the shelf" existing web services to be paid depending on usage. It will be accomplished by "assembling" different functionalities provided by different and remote ASP (Application Service Providers). In this scenario each company will provide a value that is complementary to reach the final required service: Web-Form Application and Email Translation and Fax Forwarding as shown in Figure 7. 


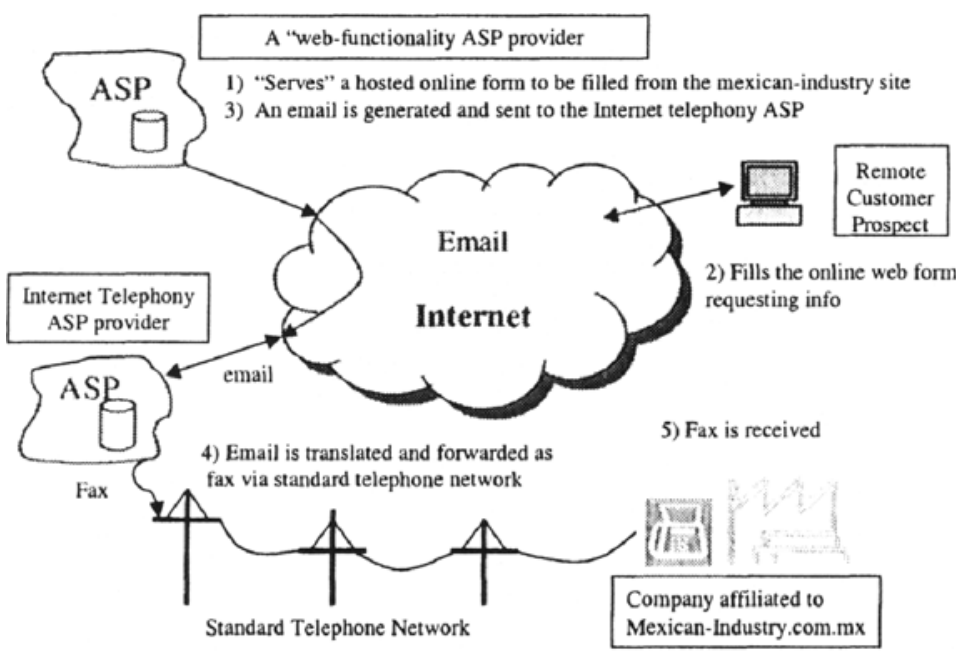

Figure 7 - "Off the shelf" e-Fax communication

\section{CONCLUSION}

Information technologies can support the development of e-services that support brokerage processes in Virtual Enterprises. In this paper a systematic methodology has been defined in order to develop e-services, which have major impacts on the VEB operations. These e-services also must offer a value added to customers that are looking for brokerage services. The proposed methodology allows VEBs, not only to identify new services concepts, but also to design and implement potential ideas into automated services. Four examples of e-services are described that are the results of applying the methodology and assuring that they will impact the performance of the VEB services.

\section{ACKNOWLEDGMENTS}

The authors gratefully acknowledge the contribution to this work of the Masters students on Global e-Management (GEM) at the EGADE business school, from the Monterrey Institute of Technology (ITESM).

\section{REFERENCES}

1. Hartman and Sifonis. "Net ready: Strategies for success in e-conomy”, McGraw Hill. N.Y., 2000.

2. Gunasekaran A. and McGaughey R. "Information technology/information systems in 21 st century manufacturing", International Journal of Productions Economics, 2002. pp. 1-6.

3. Kanet J., Faisst W. and Mertens P. "Application of information technology to a virtual enterprise broker: The case of Bill Epstein". International Journal of Production Economics, 1999. pp. 23-32.

4. Mejia R. and Molina A. "Virtual Enterprise Broker: Process, Methods and Tools", 3rd IFIP Working Conference on Infrastructures for Virtual Enterprises (PRO-VE'02), Portugal, 2002. pp. 81-90.

5. Xu W., Wei Y. and Fan Y. "Virtual Enterprise and its intelligence management", Institute of Policy and Management, Chinese Academy of Sciences, Computers \& Industrial Engineering, 2002. pp. 199-205

6. VIC Web Site (2001), (http://www.mexican-industry.com) 